\title{
Mechanical Tension Promotes the Osteogenic Differentiation of Rat Tendon-derived Stem Cells Through the Wnt5a/Wnt5b/JNK Signaling Pathway
}

\author{
Xiangzhou Liu ${ }^{a}$ Wan Chen ${ }^{a}$ You Zhou ${ }^{a}$ Kanglai Tang ${ }^{a}$ Jiqiang Zhang ${ }^{b}$ \\ aDepartment of Orthopaedics, Southwest Hospital, Third Military Medical University, Chongqing, China, \\ ${ }^{b}$ Department of Neurobiology, Third Military Medical University, Chongqing, China
}

\section{Key Words}

Rat tendon-derived stem cells (rTDSCs) • Osteogenic differentiation $\bullet$ Wnt5a $\bullet$ Wnt5b • JNK

\begin{abstract}
Background/Aims: Tendinopathy is a common sports injury that is manifested by the heterotopic ossification of tendon tissue. Tendon stem cells (TSCs) are prone to osteogenic differentiation under excessive tension. The underlying mechanisms remain poorly understood. Methods: Uniaxial mechanical tension (UMT) served to stretch rat tendon-derived stem cells (rTDSCs) at $8 \%$ elongation (frequency: $1 \mathrm{~Hz} ; 48,60$, or 72 hours). Results: The osteogenic differentiation of rTDSCs appeared after UMT along with increased mRNA expression of the osteogenic genes Runx2, Dlx5, Alpl, and Col1a1 and increased Runx2 protein expression. Wnt5a, Wnt5b and P-JNK protein levels were also upregulated after UMT stimulation. The inhibition of JNK expression by SP600125 and JNK1-shRNA decreased UMT-induced Runx2 protein expression, and the activation of JNK expression by anisomycin and JNK1-CDNA increased UMT-induced Runx2 protein expression. When shRNA knocked down Wnt5a and Wnt5b expression in rTDSCs, the induction of Runx2 and P-JNK expression by UMT was reduced. The inhibition of Runx2 expression could be rescued by the activation of JNK expression by anisomycin. Conclusion: UMT induced the osteogenic differentiation of rTDSCs via the Wnt5a/Wnt5b/JNK signaling pathway. Accordingly, this pathway may influence the heterotopic ossification of tendon tissue subjected to excessive tension.
\end{abstract}

Dr. Kanglai Tang,

and Dr. Jiqiang Zhang,

\section{KARGER 125}

Department of Orthopaedics, Southwest Hospital, Third Military Medical University, No. 29 Gaotanyan Road, Chongqing, 400038 (China) and Department of Neurobiology, Third Military Medical University, No30 Gaotanyan Road, Chongqing, 400038 (China) E-Mail tangkanglai@Hotmail.com, E-Mail zhangjqtmmu@yahool.com 


\section{Cellular Physiology Cell Physiol Biochem 2015;36:517-530

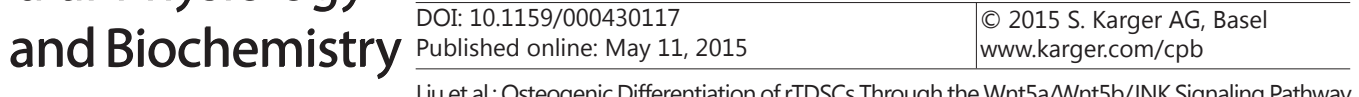

\section{Introduction}

Tendinopathy is one of the main injuries treated in orthopedics and sports medicine. The main pathological change in tendinopathy is heterotopic ossification and adipose tissue formation in tendon tissue [1]. The majority of scholars believe that tendon fiber injury caused by excessive tension loading is the leading cause of tendon disease. Bi et al. have demonstrated that tendon stem cells (TSCs) are the precursors of tendon cells and are capable of differentiating into multiple lineages and possess multiple differentiation capacity [2]. Gulotta et al. found that TSCs have a biological function similar to that of mesenchymal stem cells (MSCs) or bone marrow mesenchymal stem cells (BMSCs) and that they are directly involved in and play a critical role in the regeneration and repair of tendon micro-damage $[3,4]$,Chen et al. found that platelet-rich plasma combined with TSCs is potentially effective for the treatment of tendinopathy [5]. Morphological changes in TSCs may influence the direction of cell differentiation. An in vitro study found that under $4 \%$ mechanical tension, TSCs mainly differentiated into tendon cells and were involved in the regeneration and repair of tendon tissue; under $8 \%$ tension, TSCs differentiated into non-tendon cells (adipose cells, chondrocytes and osteoblasts) [6]. However, the mechanism by which mechanical tension induces morphological and cytoskeletal changes in TSCs that lead to osteogenic differentiation is unclear. The c-Jun amino N-terminal protein kinases (JNKs) are involved in the phosphorylation of c-Jun at the N-terminal Ser-63 and Ser-73 sites in the transcriptional activation domain $[7,8]$. Activated JNK exhibits mechanical sensitivity and is associated with osteogenic differentiation and bone formation [9-12], activation of the JNK signaling pathways upstream and downstream effectors upregulate the expression of the osteogenic gene Runx2 [13-16]. Tetsunaga et al. found that SW1353 chondrosarcoma cells increased Runx2 expression in response to mechanical tension via the JNK signaling pathway [17]. The Wnt/PCP (planar cell polarity) signaling pathway regulates the JNK signaling pathway. The activation of Wnt signaling pathway modulates the proliferation and differentiation of osteoblasts [18-20]. In vertebrates, the Wnt5a, Wnt5b, and Wnt11 ligands, as well as other ligands, activate Frizzled receptors and a co-receptor, Ror2, which then activate the G protein and Dvl within a trimetric complex that assembles into a Dvl/effector complex. The Dvl-Rac1 complex then activates JNK to regulate the cytoskeleton and cell adhesion [21-23]. Rac1 is a small molecule GTP enzyme that is involved in regulating cell differentiation and the osteogenic differentiation of MSCs [24, 25]. Ror2, a receptor tyrosine kinase (RTK)-related receptor, binds to Wnt5a and Wnt5b and is Dvl or $\mathrm{Ca}^{2+}$ dependent. Ror2 is associated with osteogenic differentiation and JNK activation [26-29]. A recent study by Park et al. found that the JNK signaling pathway promotes the osteogenic differentiation of mouse MC3T3-E1 cells [30]. Charoenpanich and others have recently shown that the Wnt5b signaling pathway is associated with the osteogenic differentiation of human mesenchymal stem cells (hMSC) induced by mechanical tension [31]. However, it is unclear whether the non-canonical WntJNK signaling pathway regulates the mechanical stress-induced osteogenic differentiation of stem cells. We speculated that uniaxial mechanical tension (UMT) may induce the osteogenic differentiation of rat tendon-derived stem cells (rTDSCs) through the Wnt5a/b/Ror2/Rac1/ JNK signaling pathway. This study aimed to explore whether UMT elicited the osteogenic differentiation of rTDSCs and to analyze the contribution of the Wnt5a/Wnt5b/JNK signaling pathway to the UMT-induced osteoblast differentiation of rTDSCs.

\section{Materials and Methods}

Isolation and culture of rTDSCS

rTDSCs were isolated as previously described [32]. Four 6-week-old male Sprague-Dawley rats weighing 150-200 g were used. The intact Achilles tendons were excised, the peritendinous tissues were removed, and the mid-substance tissues were minced and digested with type I collagenase (Sigma-Aldrich, St. Louis, MO, USA) for $2.5 \mathrm{~h}$ at $37^{\circ} \mathrm{C}$. To yield a single-cell suspension, the tissues were passed through a 70 


\section{Cellular Physiology Cell Physiol Biochem 2015;36:517-530

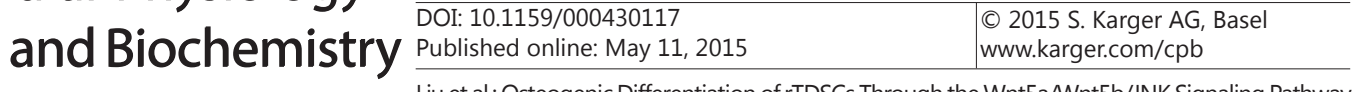

$\mu \mathrm{m}$ cell strainer (Becton Dickinson, NJ, USA). The released rTDSCs were resuspended in growth medium (GM) containing Dulbecco's Modified Eagle Medium (DMEM), $100 \mathrm{mg} / \mathrm{ml}$ streptomycin, $100 \mathrm{U} / \mathrm{ml}$ penicillin, and 10\% fetal bovine serum (all from HyClone, Logan City, Utah, USA). To enable colony formation, the isolated rTDSCs were cultured at $37^{\circ} \mathrm{C}$ in a $5 \% \mathrm{CO}_{2}$ atmosphere. Three days after initial plating, the rTDSCs were washed once with PBS to remove the non-adherent cells. At day 7, the rTDSCs were trypsinized as passage $0\left(\mathrm{P}_{0}\right)$.

\section{Identification of $r$ TDSCS}

Immunofluorescence. The rTDSCs were fixed with $4 \%$ paraformaldehyde for 30 minutes, washed twice with PBS, and blocked in $2 \%$ goat serum for 1 hour. After washing with PBS, the rTDSCs were incubated with antibodies against nucleostemin, Oct-4 and Nanog (all from Santa Cruz Biotechnology, Santa Cruz, CA, USA) for 3 hours at room temperature. After washing with PBS, a Cy3-conjugated secondary antibody (Santa Cruz) was applied for 30 minutes. After counterstaining with DAPI (Sigma-Aldrich, St. Louis, MO, USA), the rTDSCs were visualized under a fluorescence microscope (Olympus IX71, Tokyo, Japan).

Flow cytometry assay. rTDSCs $\left(2 \times 10^{6}\right.$ at $\left.\mathrm{P}_{2}\right)$ were centrifuged at $12,000 \mathrm{~g}$ for 5 minutes; the supernatant was discarded, and the cells were re-suspended in $300 \mu \mathrm{l}$ of PBS. A total of $3.0 \times 10^{5}$ events were counted for each sample. Antibody $(1 \mu \mathrm{g})$ was added to each sample. The samples were then incubated for 30 minutes at room temperature, washed with PBS, and centrifuged at 12,000 g for 5 minutes; the supernatant was subsequently discarded. The cells in each sample were re-suspended in $300 \mu \mathrm{l}$ of PBS and analyzed by flow cytometry (Becton Dickinson, San Jose, CA, USA). The following antibodies were used: anti-CD31 (ab33858; Abcam, Cambridge, UK), anti-CD90 (ab33694; Abcam, Cambridge, UK), anti-CD44 (ab23396; Abcam, Cambridge, UK) and anti-CD34 (sc-7324, Santa Cruz Biotechnology, Santa Cruz, CA, USA).

\section{Osteogenic differentiation assays}

rTDSCs were cultured in GM. After 24 hours, the GM was carefully aspirated from each well, and $2 \mathrm{ml}$ of induction medium was added (GM supplemented with $10 \mathrm{nM}$ dexamethasone, $2 \mathrm{mM}$ glutamine, $5 \mathrm{mM}$ $\beta$-glycerophosphate, $50 \mathrm{mM}$ ascorbate, $100 \mathrm{U} / \mathrm{ml}$ penicillin, and $10 \mu \mathrm{g} / \mathrm{ml}$ streptomycin; all from Cyagen Biosciences Inc, Santa Clara, CA, USA). The cells were fed with fresh induction medium every 3 days. After 21 days, the mRNA expression of Runx2, DIX5, wnt5a and wnt5b was determined. For Alizarin Red staining, the induction medium was removed, and the wells were rinsed twice with PBS. The cells were then fixed with 2 $\mathrm{ml}$ of $4 \%$ formaldehyde solution for 30 minutes, rinsed twice with PBS and stained with $1 \mathrm{ml}$ of Alizarin Red working solution (Cyagen Biosciences Inc., Santa Clara, CA, USA) for 5 minutes. The wells were rinsed twice with PBS, and the cells were visualized under a light microscope (Olympus BX51, Tokyo, Japan).

hRNA and JNK1-cDNA). After fresh DMEM was added, the transfected rTD

\section{Biochemical agents and ShRNA and CDNA transfections}

rTDSCs were exposed to $10 \mu \mathrm{M}$ SP600125 (ab120065; Abcam, Cambridge, UK) or $50 \mu \mathrm{M}$ anisomycin (CAS 22862-76-6; Santa Cruz Biotechnology, Santa Cruz, CA, USA) for 30 minutes before UMT stimulation. Recombinant lentiviral vectors expressing Wnt5b-shRNA and JNK1-shRNA were constructed by BioWit Technologies Co., Ltd (Shenzhen, China); the shRNAs were inserted into a lentiviral expression vector that contains the GFP (green fluorescent protein) gene as a reporter. The recombinant vectors were cotransfected into 293T cells; the titer of the recombinant Wnt5b-shRNA lentivirus was $8 \times 10^{8}$ infectious units per $\mathrm{mL}$, and the titer of the recombinant JNK1-shRNA lentivirus was $1 \times 10^{8}$ infectious units per $\mathrm{mL}$. The shRNA sequences targeting Wnt5b are as follows: forward: $5^{\circ}$-GGA CAA CTT GTC TGT CTT TGG TTC AAG AGA CCA AAG ACA GAC AAG TTG TCC TTT TTT CTC GAG G-3', reverse: 5'-GAT CCC TCG AGA AAA AAG GAC AAC TTG TCT GTC TTT GGT CTC TTG AAC CAA AGA CAG ACA AGT TGT C C-3'. The shRNA sequences targeting JNK1 are as follows: forward: 5'-CTG ACA AGC AGT TAG ATT CAA GAG ATC TAA CTG CTT GTC AGG GAT CTT TTT TCT CGA GG-3', reverse: 5'-GAT CCC TCG AGA AAA AAC TGA CAA GCA GTT AGA TCT CTT GAA TCT AAC TGC TTG TCA GGG ATC-3'. A recombinant lentiviral vector expressing Wnt5a-shRNA was constructed by GeneChem Co. Ltd (Shanghai, China). Wnt5a-shRNA was introduced into a lentiviral expression vector that expressed the GFP reporter gene. This recombinant vector was co-transfected into 293T cells, and the titer of the recombinant lentivirus was $3 \times 10^{8}$ infectious units per mL. The shRNA sequences targeting Wnt5a are as follows: forward: 5'-CCG GAA CCC TGT TCA GAT GTC AGA ACT CGA GTT CTG ACA TCT GAA CAG GGT TTT-3' and reverse: 5'-AAT TCA AAA ACC CTG TTC AGA TGT CAG AAC TCG AGT TCT GAC ATC TGA ACA GGG 


\section{Cellular Physiology Cell Physiol Biochem 2015;36:517-530

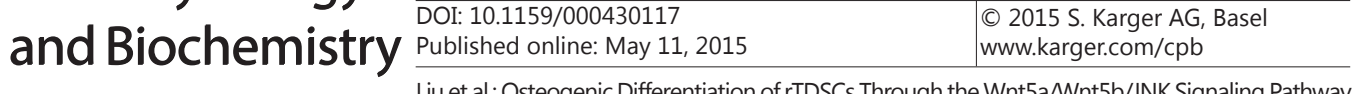

TT-3'. A scrambled shRNA vector was constructed by BioWit Technologies Co., Ltd (Shenzhen, China); the scrambled shRNA sequence (TTC TCC GAA CGT GTC ACG T) was used as the universal negative control [3335] (shRNA-control) throughout the study. Rat JNK1-cDNA was introduced into the lentiviral vector pLVXrJNK1-mCMV-ZsGreen by BioWit Technologies Co., Ltd (Shenzhen, China) for overexpression. The pLvxmCMV-ZsGreen vector was used as the scrambled control (cDNA-control). The titer of the recombinant lentivirus was $1 \times 10^{8}$ infectious units per $\mathrm{mL}$. The cDNA sequences targeting JNK1 are as follows: forward: 5'-CAT CCA CGC TGT TTT GAC CTC CAT AGA AGA CAC CGA CTC TAC TAG AGG ATC TAT TTC CGG-3', reverse: 5'-TGA ATT CCT CGA GAC TAG TTC TAG AGC GGC CGC CAC CAT GAG CAG AAG TAA ACG TGA CAA-3'. rTDSCs were plated into 6 -well plates at $1 \times 10^{5}$ cells per well and were maintained in DMEM overnight. When the cells reached $50 \%$ confluence, they were infected with lentiviral particles for 24 hours at a multiplicity of infection (MOI) of 40 (Wnt5a-shRNA, Wnt5b-shRNA, JNK1-shRNA and JNK1-cDNA). After fresh DMEM was added, the transfected rTDSCs cells were subcultured at an appropriate density. After an additional 3 days in culture, the infection efficiency was determined by counting GFP-expressing cells by fluorescence microscopy (Olympus BX51, Tokyo, Japan) and by real-time PCR (RT-PCR) and Western blot analysis.

\section{Uniaxial mechanical tension}

rTDSCs at $\mathrm{P}_{2}$ were plated at a density of $2 \times 10^{4}$ cells $/ \mathrm{cm}^{2}$ in $2.5 \mathrm{ml}$ of GM in 14 silicone culture dishes (B-Bridge International, Inc., Cupertino, CA, USA). The bottom of each dish was coated with type I collagen (Sigma-Aldrich, St. Louis, MO, USA). The cells were then incubated for 24 hours at $37^{\circ} \mathrm{C}$ in $5 \% \mathrm{CO}_{2}$ to facilitate cell adhesion and were allowed to reach 70-80\% confluence. Seven silicone dishes (UMT group) were then stretched in an intelligent bionic uniaxial mechanical tension system (YAHO/XBQX-2000, Yahong Technologies Co., Ltd., Chongqing, China). The settings for the UMT were the following: 1 HZ frequency, $8 \%$ elongation, 4 hours at each tension, and a 4 hour interval. The other silicone dishes served as the nonloading control (NC) group.

\section{Cytoskeletal staining}

rTDSCs were plated in silicone dishes at a concentration of $2 \times 10^{4}$ cells $/ \mathrm{cm}^{2}$. When the rTDSCs had attached to the base film, UMT was applied to the silicone dishes at a frequency of $1 \mathrm{~Hz}$ with $4 \%$ or $8 \%$ elongation for 4 hours; each tension interval was 4 hours. After a total of 24 hours of UMT, rTDSCs were washed twice with PBS, fixed with 4\% paraformaldehyde for 20 minutes and then washed twice with PBS. rTDSCs were subsequently treated with $0.3 \%$ Triton X-100 for 10 minutes, stained with $5 \mu \mathrm{g} / \mathrm{ml}$ rhodaminephalloidin (p2141, Sigma-Aldrich, St. Louis, MO, USA) at room temperature for 1 hour and washed twice with PBS. rTDSCs were exposed to DAPI for 10 minutes to stain the nuclei and were washed twice with PBS. rTDSCs were observed using a confocal microscope (Carl Zeiss, Jena, Germany).

\section{Alkaline phosphatase staining}

rTDSCs that had been treated with 8\% UMT for 72 hours and rTDSCs in the NC group were washed twice with PBS and fixed with 4\% paraformaldehyde for 8 minutes. The rTDSCs were incubated with an NBT/BCIP solution (Sigma-Aldrich, St. Louis, MO, USA) for 30 minutes at room temperature and were washed twice with PBS. rTDSCs were visualized using a light microscope (Olympus BX51, Tokyo, Japan).

\section{Real-time PCR}

Total RNA was isolated using TRIzol reagent (Takara, Dalian, China). After total RNA was reversetranscribed, real-time PCR (RT-PCR) was performed using the Bio-Rad iCycler IQ system (Bio-Rad, CA, USA). Relative gene expression levels were calculated using the $2^{-\Delta \Delta \mathrm{Ct}}$ formula; Gapdh was used as the internal control. The data were expressed as the fold change after 48, 60 and 72 hours of UMT compared with the NC group. At least three independent experiments were performed. The primer sequences are as follows: Gapdh (NM_017008.4) forward: 5'-TGA CTT CAA CAG CAA CTC-3', reverse: 5'-TGT AGC CAT ATT CAT TGT CA-3'; Wnt5a (NM_022631.1) forward: 5'-TTC CAA GTT CTT CCT AAT-3', reverse: 5'-TAT ACT TCT GAC ATC TG AA-3'; Wnt5b (NM_001100489.1) forward: 5'-GCA GGG TCA TGC AGA TAG G-3', reverse: 5'-CGG TAG CCA TAC TCC ACG ACG T-3'; Ror2 (NM_001107339.1) forward: 5'-CTT CCC ACT CTG AAA GGC TAC TT3', reverse: 5'-TAC GAC AGG GGC ATC ATT CTT C-3'; Rac1 (NM_134366.1) forward: 5'-CTG CTC ATC AGT TAC ACG AC-3', reverse: 5'-GGA CGC AGT CTG TCA TAA TC-3'; Runx2 (NM_001278483.1) forward: 5'-GAA CTC AGC ACC AAG TCC TTT-3', reverse: 5'-CAG TGT CAT CAT CTG AAA TAC GC-3'; Dlx5 (NM_012943.1) 


\section{Cellular Physiology Cell Physiol Biochem 2015;36:517-530

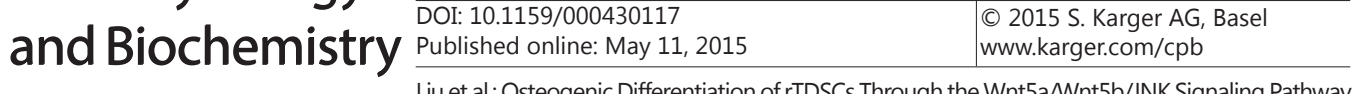

forward: 5'-CTT ATG CGG A CTA CGG CTA CG-3' ${ }^{\prime}$, reverse: 5'-CCT GGG TTT ACG AAC TTT CTT TG-3'; Col 1a1 (NM_053304.1) forward: 5' -TGG TGA GAC GTG GAA ACCT G-3' ${ }^{\prime}$, reverse: 5' ${ }^{\prime}$-CTT GGG TCC CTC GAC TCC TA-3'; and Alpl (NM_013059.1) forward: 5'-GGC ACC ATG ACT TCC CAG AA-3', reverse: 5'-CAC CGT CCA CCA CCT TGT AA-3'.

Western blotting

The following primary antibodies were used: anti-Runx2 (1:1000; ab23981, Abcam, Cambridge, UK), anti-Wnt5a (1:400; ab110073, Abcam, Cambridge, UK), anti-Wnt5b (1:1000; GTX47175, GeneTex, Irvine, CA, USA), anti-JNK1/JNK2/JNK3 (1:1000; ab179461, Abcam, Cambridge, UK), anti-JNK1/JNK2/JNK3 (phospho-T183/T183/T221; 1:1000; ab124956, Abcam, Cambridge, UK), anti-JNK1 (1:1000; ab199380, Abcam, Cambridge, UK), anti-JNK1 (phospho-T183; 1:1000; ab47337, Abcam, Cambridge, UK), GAPDH (1:1000; Pierce BIC, USA), and $\beta$-tubulin (1:1000; loading control; Pierce). rTDSCs were lysed in lysis buffer (50 mM Tris- $\mathrm{HCl}$, pH 8.0,1\% Triton X-100, 0.5\% sodium deoxycholate, 1 mM EDTA, $150 \mathrm{mM} \mathrm{NaCl}$, and $0.1 \%$ sodium dodecyl sulfate) with proteinase inhibitors (Thermo Fisher Scientific Inc., Rockford, IL, USA). Samples (30 $\mu \mathrm{g} / \mathrm{lane}$ ) were resolved by SDS-PAGE and transferred onto polyvinylidene difluoride membranes. The results were visualized using a LiCor Odyssey Imager (LI-COR Biosciences, Lincoln, NE, USA).

Statistical analysis

The NC and UMT groups were compared using a Student's t-test. The data analysis was performed using SPSS software 16.0 (SPSS Inc., Chicago, IL, USA). The statistical significance level was $\mathrm{P}<0.05$.

\section{Results}

Identification and osteogenic differentiation of rTDSCs

The shape of the rTDSCs from $\mathrm{P}_{0}$ to $\mathrm{P}_{3}$ was similar to elongated fibroblasts (Fig. 1A). The flow cytometry analysis showed that rTDSCs exhibited positive staining for the MSC marker CD90 but negative staining for CD44, the hematopoietic cell marker CD34 and the endothelial cell marker CD31 (Fig. 1B). The immunofluorescence analysis demonstrated that the stem cell markers Oct-4, Nanog and nucleostemin were expressed in rTDSCs (Fig. 1C). rTDSCs have the capacity to differentiate into osteoblasts. After 21 days of osteogenic induction, the RT-PCR analysis revealed that the mRNA expression of Runx2 and DIX5 was higher in the osteogenic media (Osteo-M) group than in the GM group (Fig. 1D). Mineralized calcium deposits were observed by Alizarin Red staining after 21 days of osteogenic induction; however, no mineralized calcium deposits were found in the GM group (Fig. $1 \mathrm{E})$. Compared to the GM group, the mRNA expression of Wnt5a and Wnt5b was higher in the Osteo-M group (Fig. 1D), indicating that Wnt5a/Wnt5b may be associated with the osteogenic differentiation of rTDSCs.

UMT induced the osteogenic differentiation of rTDSCS

UMT for 48,60 , or 72 hours at $8 \%$ elongation and a frequency of $1 \mathrm{HZ}$ increased the mRNA expression of the osteogenic genes Runx2, Dlx5, Alpl, and Col1a1 in rTDSCs compared with the NC group (Fig. 2A-C). Similarly, Runx2 protein expression in rTDSCs was higher after 48,60 , or 72 hours of UMT compared with the NC group (Fig. 2D). The alkaline phosphatase staining level in the 72-hour UMT group was higher than that in the NC group (Fig. 2E).

Upregulation of Wnt5a, Wnt5b, Ror2, and Rac1 in rTDSCs after UMT

UMT for 48, 60, or 72 hours increased the mRNA expression of non-canonical Wnt5a/ Wnt5b in rTDSCs as well as that of Ror2 and Rac1 compared with the NC group (Fig. 3A-C). The Wnt5a and Wnt5b protein levels were higher in rTDSCs exposed to 48 and 60 hours of UMT compared with the NC group (Fig. 3D-E). 


\section{Cellular Physiology Cell Physiol Biochem 2015;36:517-530 \begin{tabular}{ll|l} 
and Bi 10.1159/000430117 & $\begin{array}{l}\text { O 2015 S. Karger AG, Basel } \\
\text { www.karger.com/cpb }\end{array}$ \\
\hline
\end{tabular}

Fig. 1. Identification and osteogenic differentiation of rTDSCs. (A) Shape of rTDSCs from $\mathrm{P}_{0}-\mathrm{P}_{3}$. Scale: $100 \mu \mathrm{M}$. (B) rTDSCs were stained for MSC markers (CD90 and CD44), an endothelial cell marker (CD31), and a hematopoietic stem cell marker (CD34) by flow cytometry; the positive population was enclosed by the line. (C) Expression of the stem cell markers Nanog, Oct-4 and nucleostemin (red dot). Bar: $10 \mu \mathrm{m}$. (D) Osteogenic differentiation of rTDSCs. After rTDSCs were cultured in growth medium (GM) and osteogenic medium (Osteo-M) for 21 days, the mRNA expression levels of Runx2, DIX5, Wnt5a and Wnt5b were determined ( $\mathrm{n}=3$; ${ }^{* *} \mathrm{P}$ $<0.01$ ). (E) Mineralized calcium deposits. rTDSCs were cultured in GM and Osteo-M for 21 days and stained with Alizarin Red (Scale: $50 \mu \mathrm{m}$ ).
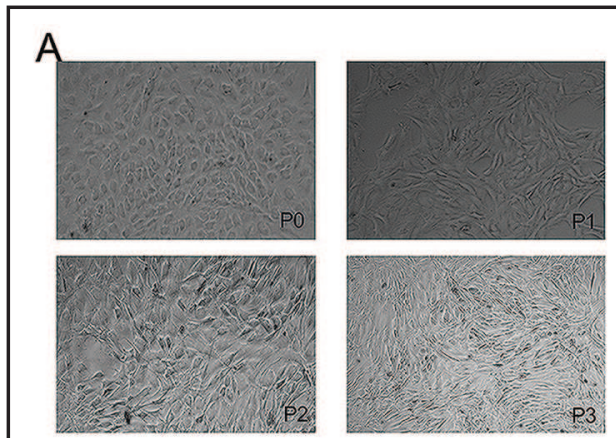

B
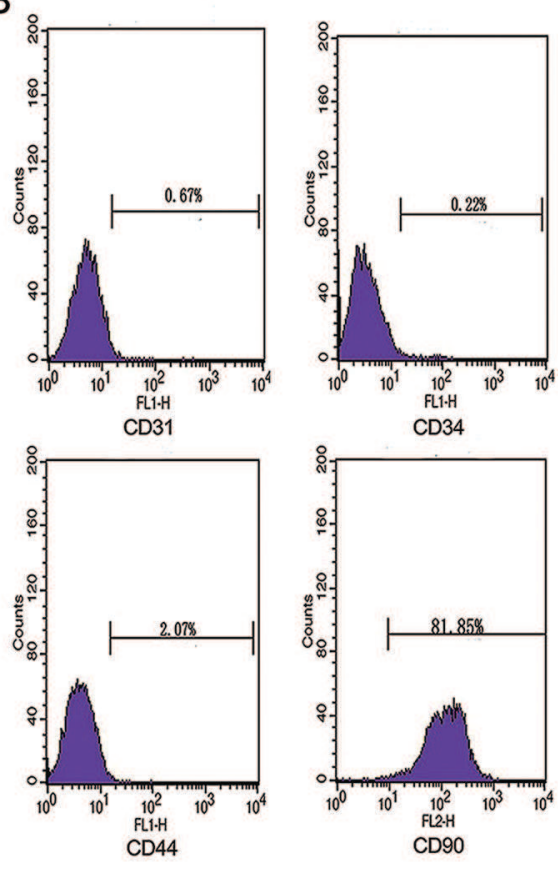
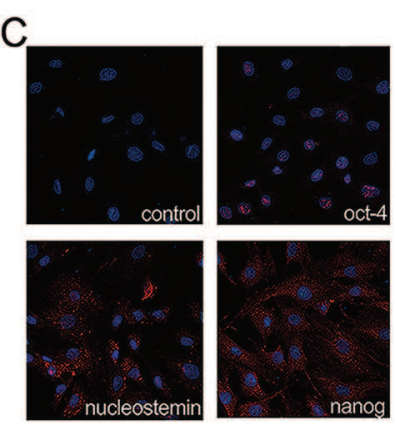

D

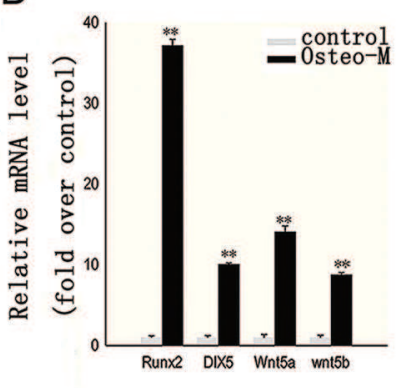

E

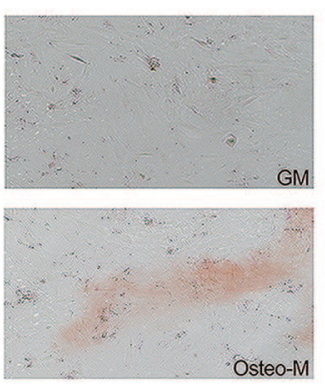

Regulation of Runx2 expression by Wnt5a/Wnt5b in rTDSCs after UMT

Wnt5a/Wnt5b mRNA (Fig. 4A-B) and protein (Fig. 4C-D) expression was significantly decreased in rTDSCs after transfection with Wnt5a-shRNA and Wnt5b- shRNA for 3 days compared with the shRNA-control group. After 60 hours of UMT, Runx2 protein expression was lower in the $8 \%+W n t 5 a-s h R N A$ and $8 \%+W n t 5 b$-shRNA groups compared with the $8 \%+$ +shRNA-control group (Fig. 4E).

UMT induced cytoskeletal changes and regulated Runx2 expression in rTDSCs through JNK

In rTDSCs exposed to 8\% elongation, actin was depolymerized and clearly broken, and the mean fluorescence intensity was decreased compared with the NC group and the $4 \%$ elongation group, suggesting that irreversible cytoskeletal changes had occurred (Fig. 5A). After 48 and 60 hours of UMT, P-JNK1/JNK2/JNK3 levels were higher in the UMT groups than in the NC group (Fig. 5B). To verify whether JNK regulated UMT-induced Runx2 expression, rTDSCs were treated with the JNK inhibitor SP600125 and the JNK activator anisomycin for 30 minutes before UMT induction. SP600125 decreased Runx2 mRNA expression, and anisomycin increased Runx2 mRNA expression in rTDSCs after UMT induction for 48 and 60 hours compared with the $8 \%+$ no drug group (8\%+Veh) (Fig. 5D). After UMT for 60 hours, Runx2 protein expression was higher in the $8 \%+$ Veh group than in the NC group, and Runx2 protein expression was higher in the anisomycin group than in the $8 \%+$ Veh group; however, SP600125 decreased Runx2 protein levels compared to the 8\%+Veh group (Fig. 5C). 


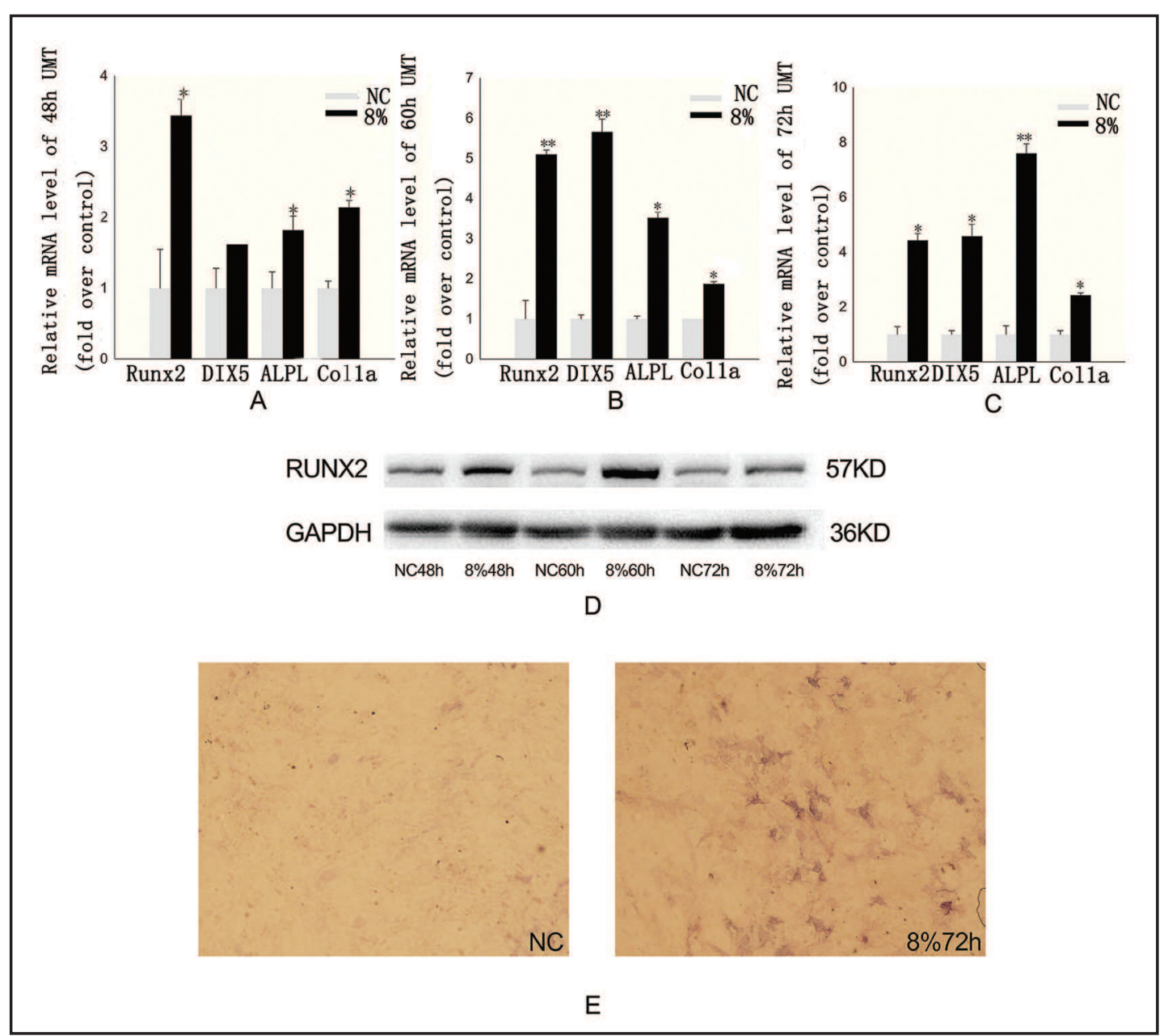

Fig. 2. UMT-induced osteogenic differentiation of rTDSCs. (A-C) The mRNA expression of osteogenic genes (Runx2, Dlx5, Alpl and Col 1a1) in rTDSCs after 8\% UMT for 48, 60, and 72 hours compared to the expression in the NC group ( $\mathrm{n}=3$; ${ }^{*} \mathrm{P}<0.05$; ${ }^{* *} \mathrm{P}<0.01$ ). (D) Runx2 protein levels in rTDSCs after UMT for 48, 60, or 72 hours relative to the NC group. Runx2 protein expression was quantitated by densitometric analysis, and GAPDH was used as the loading control. (E) ALP staining of rTDSCs after 72 hours of 8\% UMT compared to the NC group (Scale: $50 \mu \mathrm{m}$ ). NC group: non-loading control group.

JNK regulated UMT-induced Runx 2 expression in rTDSCs as confirmed by transfection with JNK1-cDNA and JNK1-shRNA

P-JNK1 expression was significantly decreased in rTDSCs after transfection with JNK1-shRNA for 3 days compared with shRNA-control (Fig. 6A).P-JNK1 expression was significantly increased in rTDSCs after transfection with JNK1-cDNA for 3 days compared with cDNA-control (Fig. 6B). After 60 hours of UMT, Runx2 protein expression was higher in rTDSCs transfected with JNK1-cDNA compared with the 8\%+cDNA-control group, and Runx2 protein expression was lower in rTDSCs transfected with JNK1-shRNA compared with the 8\%+shRNA-control group (Fig. 6C).

Wnt5a/Wnt5b regulated UMT-induced Runx2 expression in rTDSCs through JNK

To determine whether Wnt5a and Wnt5b regulated UMT-induced Runx2 expression through the JNK pathway, rTDSCs were transfected with Wnt5a-shRNA and Wnt5b-shRNA for 3 days; 30 minutes before UMT, anisomycin was added to the Wnt5a-shRNA and Wnt5bshRNA groups. The UMT-induced upregulation of Runx 2 mRNA expression was suppressed in rTDSCs transfected with shRNA-Wnt5a and shRNA-Wnt5b compared to the 8\%+shRNA- 


\section{Cellular Physiology Cell Physiol Biochem 2015;36:517-530

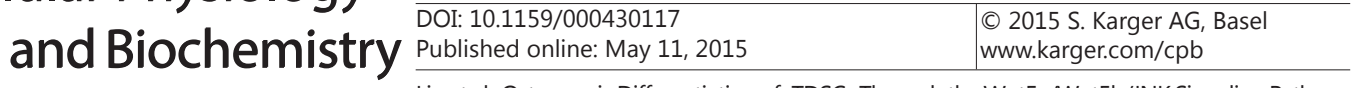

Fig. 3. UMT-induced Wnt5a, Wnt5b, Ror2 and Rac1 expression. The mRNA expression of Wnt5a, Wnt5b, Ror2 and Rac1 in rTDSCs after UMT for 48 hours (A), 60 hours (B) and 72 hours (C) relative to the NC group $\left(\mathrm{n}=3\right.$; ${ }^{*} \mathrm{P}$ $<0.05$; ${ }^{* *} \mathrm{P}<0.01$ ). Protein expression of Wnt5a (D) and Wnt5b (E) in rTDSCs after UMT for 48 and 60 hours relative to the NC groups. $\beta$-tubulin was used as the loading control. NC group: non-loading control group.

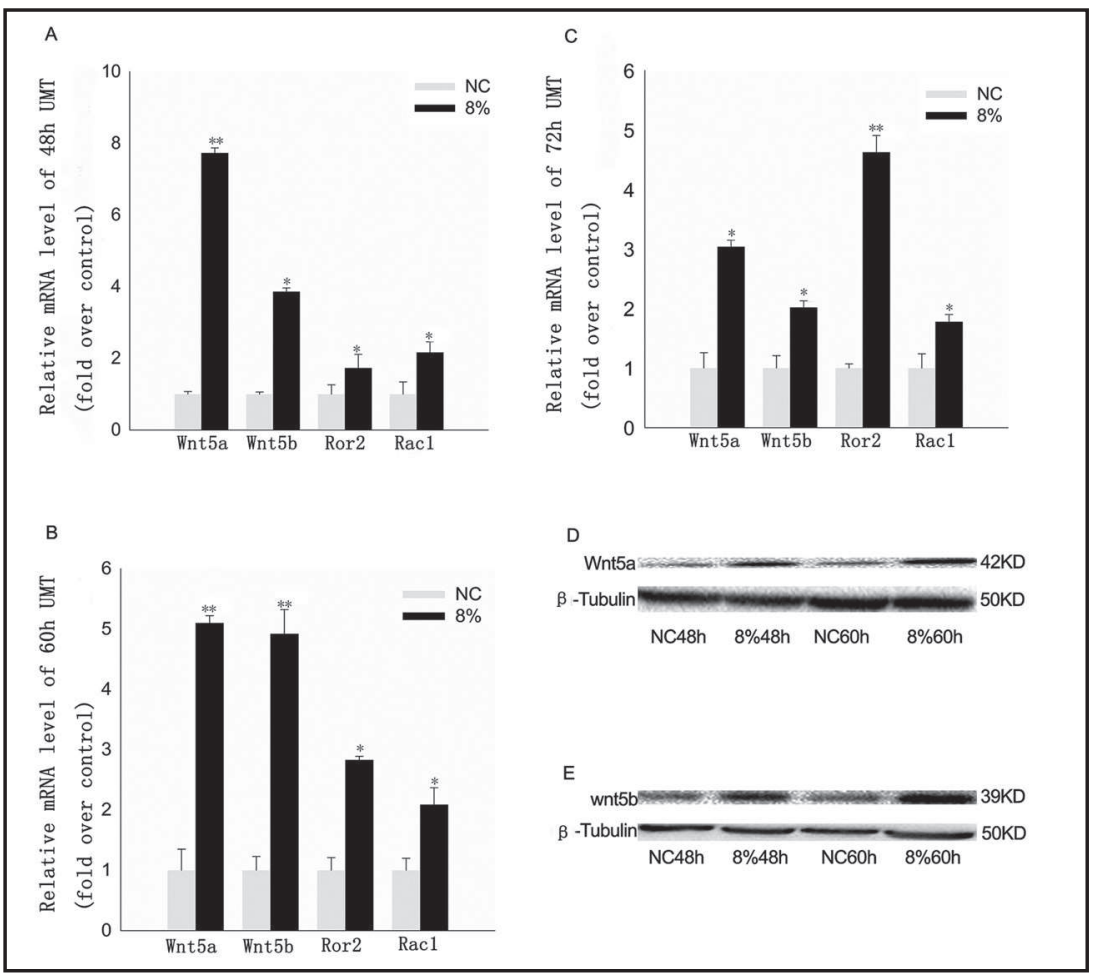

Fig. 4. Wnt5a and Wnt5b regulated the UMT-induced osteogenic differentiation of rTDSCs. The mRNA expression levels of $\mathrm{Wn}$ t5a (A) and Wnt5b (B) in rTDSCs after 3 days of shRNA treatment relative to scrambled shRNA control (shRNA-control) ( $\mathrm{n}=3$; ${ }^{*} \mathrm{P}$ $<0.05$ ). The Wnt5a (C) and Wnt5b (D) protein levels were determined by densitometric analysis relative to the shRNA-control groups. $\beta$-tubulin was used as the loading control.

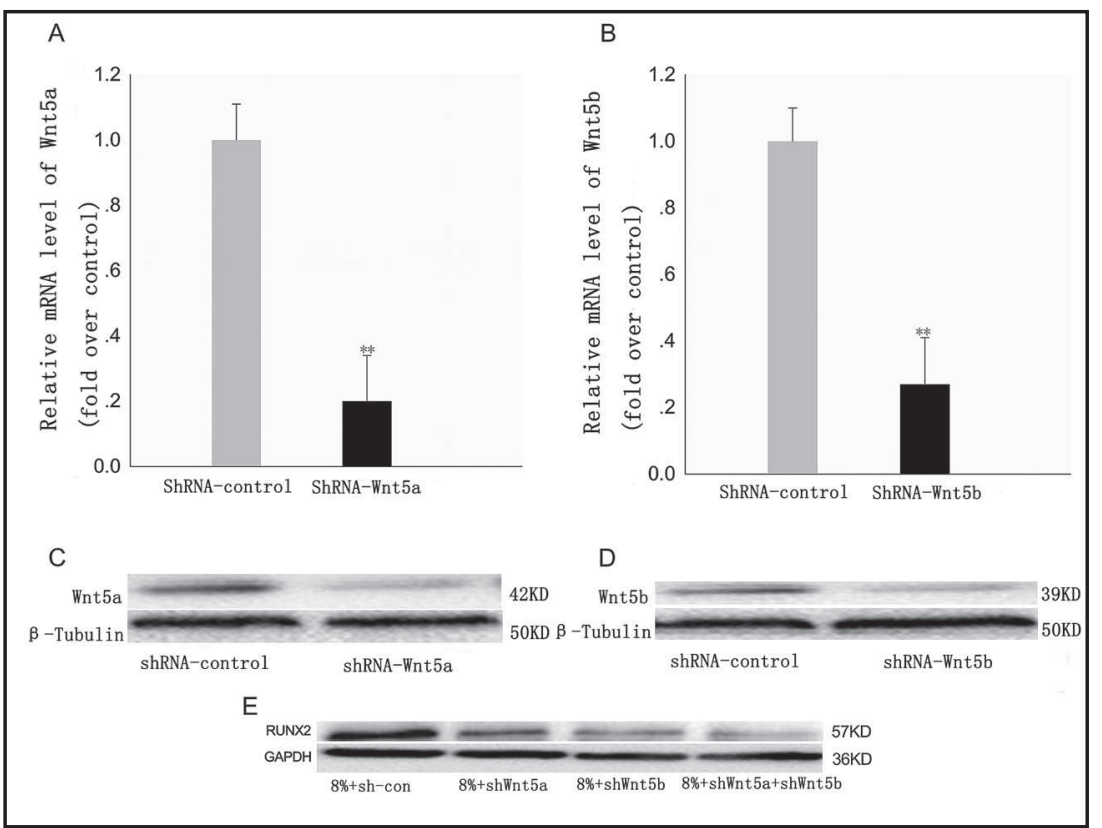

(E) 3 days after transfection with Wnt5a-shRNA, Wnt5b-shRNA and Wnt5a-shRNA+Wnt5b-shRNA, rTDSCs were treated with $8 \%$ UMT for 60 hours. The Runx2 protein levels are shown relative to the $8 \%+$ shRNA-control group. GAPDH was used as the loading control. Sh-con: shRNA-control; ShWnt5a: Wnt5a-shRNA; ShWnt5b: Wnt5b-shRNA.

control group. Runx2 mRNA expression was restored by the addition of anisomycin (Fig. 7A) .The P-JNK1 level were lower in the $8 \%+$ shRNA-Wnt5a and $8 \%+$ shRNA-Wnt 5 b groups than in the 8\%+shRNA-control group after UMT for 60 hours. The UMT-induced upregulation of P-JNK1 level was suppressed in rTDSCs transfected with Wnt5a-shRNA and Wnt5b-shRNA; 


\section{Cellular Physiology Cell Physiol Biochem 2015;36:517-530

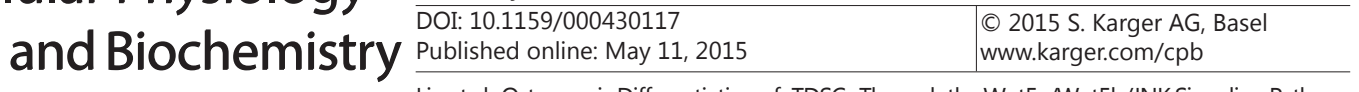

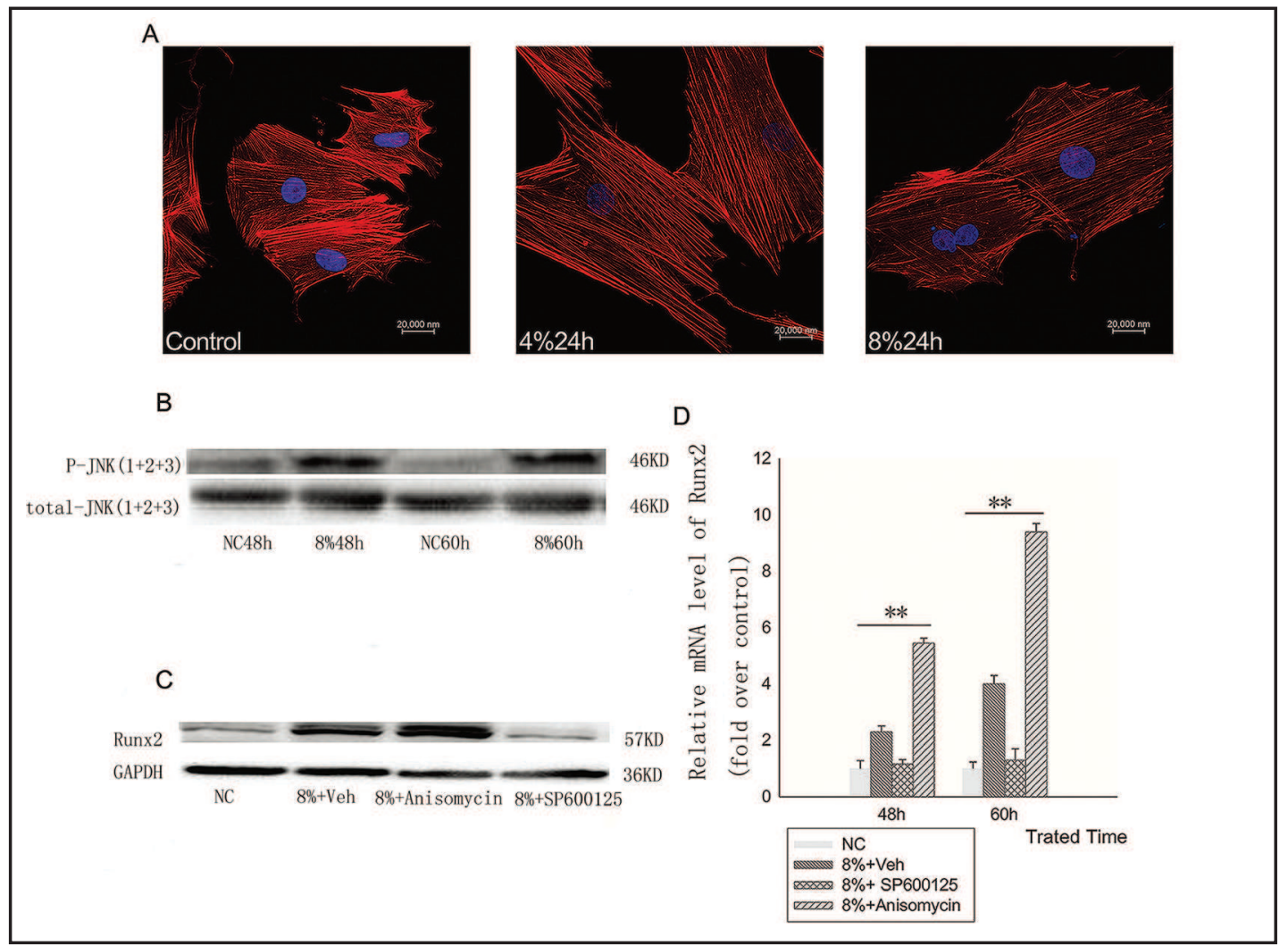

Fig. 5. UMT induced cytoskeletal changes and regulated Runx2 expression in rTDSCs through JNK. (A) rTDSCs were treated with $4 \%$ and $8 \%$ UMT for 24 hours, and the cytoskeleton was stained with rhodamine-phalloidin. Scale: $20 \mu \mathrm{m}$. (B) Western blot analysis of phosphorylated JNK (P-JNK1/JNK2/JNK3) in rTDSCs treated with UMT for 48 and 60 hours. Total-JNK1/JNK2/JNK3 was used as the loading control. (C) Before UMT, rTDSCs were treated with $10 \mu \mathrm{M}$ SP600125 or $50 \mu \mathrm{M}$ anisomycin for 30 minutes. Runx 2 mRNA expression was detected after UMT treatment for 48 and 60 hours. The data are presented as the fold change relative to the NC group ( $\mathrm{n}=3$; ${ }^{* *} \mathrm{P}<0.01$ ). (D) Runx2 protein expression in the $8 \%+V e h, 8 \%+S P 600125$ and $8 \%+$ anisomycin groups after UMT for 60 hours relative to the NC group. GAPDH was used as the loading control. NC group: non-loading control group; $8 \%+$ Veh: 8\% UMT without drug treatment.

this inhibition could be reversed by the JNK activator, anisomycin (Fig. 7B). The UMTinduced upregulation of Runx2 protein expression was suppressed in rTDSCs transfected with shRNA-Wnt5a and shRNA-Wnt5b compared to the 8\%+shRNA-control group. Runx2 protein expression was restored by the addition of anisomycin (Fig. 7C).

\section{Discussion}

Tendon tissue calcification is one of the main manifestations of tendinopathy. The majority of scholars believe that tendinopathy is caused by chronic fatigue and excessive tension loading, leading to tendon microfiber injuries. These microfiber injuries are considered an initial risk factor for tendon injuries [6,36]. In 2007, Bi et al. identified stem cells in tendon tissue [2], named TDSCs. TDSCs have the capacity for multipotent differentiation, and studies have shown that TDSCs undergo osteogenic differentiation in response to mechanical stress [37]. Lui et al. proposed that TDSCs differentiate into nontenocytes in response to mechanical stress, which may be the cause of the heterotopic ossification of tendon tissue [38]; however, the mechanisms for this remain unclear. This study aimed to investigate the mechanism of TDSC osteogenic differentiation in response to 


\section{Cellular Physiology Cell Physiol Biochem 2015;36:517-530

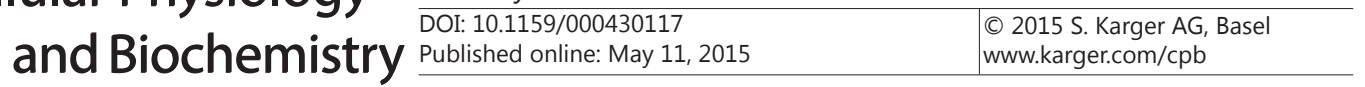

Liu et al.: Osteogenic Differentiation of rTDSCs Through the Wnt5a/Wnt5b/JNK Signaling Pathway

A

B

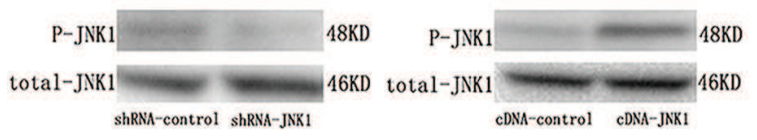

C

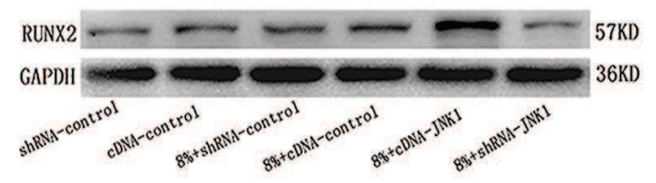

Fig. 6. JNK1 regulated the UMT-induced Runx2 expression in rTDSCs as confirmed by transfection with JNK1-cDNA and JNK1-shRNA. (A) Phosphorylated JNK1 (P-JNK1) levels in rTDSCs after transfection with JNK1-cDNA for 3 days compared with cDNA-control. (B) P-JNK1 levels in rTDSCs after transfection with JNK1-shRNA for 3 days compared with shRNA-control. Total-JNK1 was used as the loading control. (C) After 60 hours of UMT induction, Runx2 protein expression in rTDSCs transfected with JNK1-cDNA was compared with that in the $8 \%+c D N A-c o n t r o l$ group, and Runx2 protein expression in rTDSCs transfected with JNK1-shRNA was compared with that in the 8\%+shRNA-control group. GAPDH was used as the loading control.

Fig. 7. Wnt5a and $W n t 5 b$ regulated UMT-induced Runx2 expression in rTDSCs through JNK. (A) rTDSCs were transfected with Wnt5a-shRNA and Wnt5b-shRNA for 3 days; 30 minutes before UMT, anisomycin was added to the Wnt5a-shRNA and Wnt5b-shRNA groups. After UMT for 60 hours, an RTPCR analysis of Runx2 mRNA expression in rTDSCs was performed. The data are presented as the relative change compared to the $8 \%+$ shRNA-control group ( $\mathrm{n}=3$; $\left.{ }^{*} \mathrm{P}<0.05\right)$. AM: anisomycin. (B) rTDSCs were transfected with Wnt5a-shRNA and Wnt5b-shRNA for 3 days; 30 minutes before UMT, anisomycin was added to the Wnt5a-shRNA and Wnt5b-shRNA groups. Western blot of P-JNK1 after 60 hours of UMT was performed with total JNK1 as the loading control. (C) rTDSCs were transfected with Wnt5a-shRNA and Wnt5b-shRNA for 3 days; 30 minutes before UMT, anisomycin was added to the Wnt5a-shRNA and Wnt5b-shRNA groups. Western blot of RUNX2 after 60 hours of UMT was performed with GAPDH as the loading control.

A

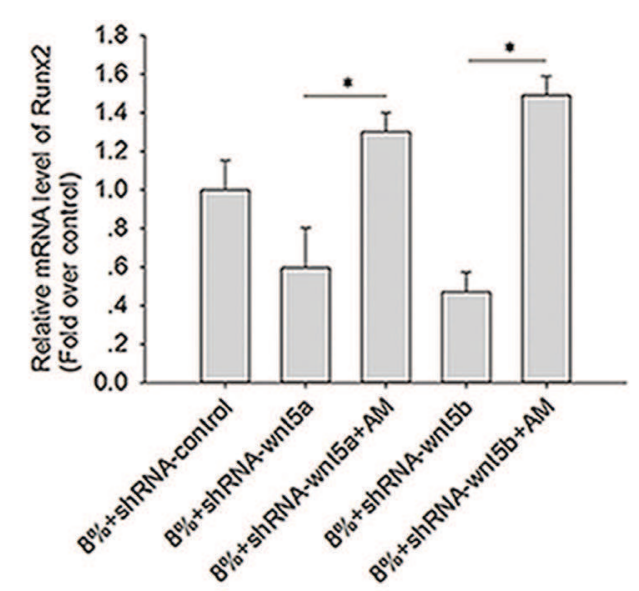

B

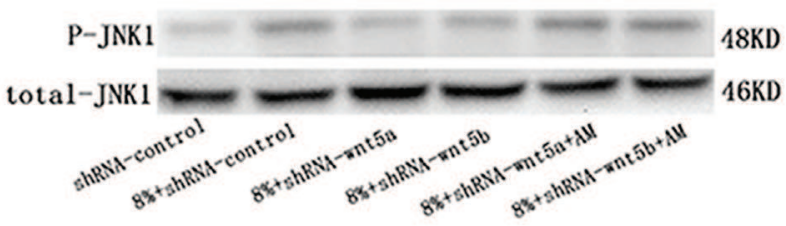

$\mathrm{C}$

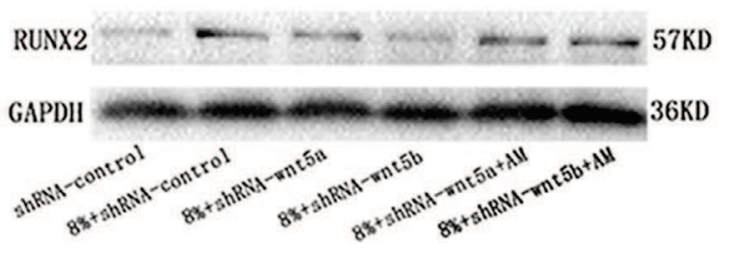

mechanical stress. Studies have shown that applying intermittent mechanical tension to MSCs (every few hours, several times a day, for several days) promotes osteogenic differentiation, whereas applying continuous mechanical tension to MSCs inhibits osteogenic differentiation 


\section{Cellular Physiology Cell Physiol Biochem 2015;36:517-530 \\ \begin{tabular}{ll|l} 
and Biochemistry & $\begin{array}{l}\text { DOI: 10.1159/000430117 } \\
\text { Published online: May 11, } 2015\end{array}$ & $\begin{array}{l}\text { C } 2015 \text { S. Karger AG, Basel } \\
\text { www.karger.com/cpb }\end{array}$ \\
\cline { 2 - 3 } & Liu et al.: Osteogenic Differentiation of rTDSCs Through the Wnt5a/Wnt5b/JNK Signaling Pathway
\end{tabular}}

[39]. In this study, osteogenic differentiation of rTDSCs was observed after intermittent UMT, as was increased mRNA expression of osteogenic genes (Runx2, Dlx5, Alpl, and Col 1a1), increased Runx2 protein expression and positive ALP staining, indicating that UMT induced the osteogenesis differentiation of rTDSCs. The current research showed that, in addition to the UMT-induced osteogenic differentiation of rTDSCs, the mRNA and protein expression of the non-canonical Wnt molecules Wnt5a/Wnt5b and the mRNA expression of Ror2 and its downstream small molecule GTP enzyme Rac1 were also elevated. Previous studies have shown that Wnt5a/Wnt5b mRNA is enhanced in stem cells under mechanical stress and that this effect is associated with osteogenic differentiation [29-31]. In a study by Shi et al., Wnt5a mRNA expression increased in rTDSCs after UMT, although Wnt5b mRNA expression was not increased [40]; these results differed from those of our study, and this discrepancy may be due to differences in the mechanical tension system or in the mechanical elongation, frequency, periodicity or duration. When shRNAs were used to knock down the expression of Wnt5a and Wnt5b, the UMT-induced osteogenic differentiation of rTDSCs was inhibited, indicating that the UMT-induced osteogenic differentiation of rTDSCs was regulated by Wnt5a and Wnt5b. Cytoskeletal changes were observed in rTDSCs after UMT. JNK is a cytoskeleton regulator, and the phosphorylation of JNK1/JNK2/JNK3 increased after UMT. The JNK activator anisomycin promoted the UMT-induced mRNA and protein expression of the osteogenic gene Runx2; the converse was observed with the JNK inhibitor SP600125. These results indicated that JNK may modulate the UMT-induced osteogenic differentiation of rTDSCs. However, anisomycin and SP600125 are not JNK-specific. Anisomycin can induce p38-MAPK, and SP600125 can inhibit a number of kinases in addition to JNK [41]. p38-MAPK also regulates stress-stimulated osteogenic differentiation [42]. The JNK gene has three isoforms, JNK1, JNK2, and JNK3; among them, JNK1 is mainly associated with osteogenic differentiation [43-45]. Therefore, we used JNK1-cDNA and JNK1-shRNA to confirm the effects of JNK signaling. JNK1-cDNA promoted UMT-induced Runx2 protein expression, whereas JNK1-shRNA inhibited Runx2 protein expression. The mechanism for this may involve force transmission, in which activated JNK and ERK1/2 convert mechanical stress to intracellular molecular events that lead to gene expression, protein synthesis, cell proliferation and cell differentiation $[46,47]$. When rTDSCs were exposed to UMT after shRNA-mediated knockdown of Wnt5a/Wnt5b, JNK activity decreased, and osteogenic differentiation was inhibited. The inhibition of osteogenesis was rescued by the JNK activator, anisomycin, or by JNK1-cDNA, indicating that Wnt5a/Wnt5b is located upstream of JNK and regulates the function of JNK. These data suggested that the effect of Wnt5a/Wnt5b on UMT-induced osteogenesis was mediated by JNK. The non-canonical Wnt pathways include Wnt/PCP, Wnt/JNK, Wnt/calcium and Wnt/Rho [11]. The Rho and JNK signaling pathways both regulate the cytoskeleton and are associated with osteogenic differentiation, but the relationship between them is unclear. Studies have shown that JNK is independent of Rho but is regulated by Rac GTPase $[48,49]$. Some scholars believe that in the process of eye development, JNK is another downstream effector of Dvl and is activated by RhoA, thereby regulating the PCP signaling pathway $[50,51]$. Studies by Wang et al. have shown that Wnt5a/JNK regulates cell movement independent of or dependent on the RhoA pathway [52]. Whether the RhoA and JNK pathways regulate each other during the UMT-induced osteogenic differentiation of TDSCs remains to be studied.

Both the current study and Wang et al. observed increased mRNA expression of adipogenic-related genes, such as PPARgamma2, during the UMT-induced osteogenic differentiation of rTDSCs [6]. However, many studies have shown that osteogenesis and adipogenesis are mutually inhibitory $[53,54]$. Whether the non-canonical Wnt signaling pathway regulates both adipogenic and osteogenic differentiation under mechanical stress awaits further study.

In conclusion, our study showed that the UMT-induced osteogenic differentiation of rTDSCs was regulated by the Wnt5a/Wnt5b/JNK signaling pathway. This work provides a potential molecular mechanism for the calcification of tendon tissue resulting from mechanical tension. 


\section{Cellular Physiology Cell Physiol Biochem 2015;36:517-530

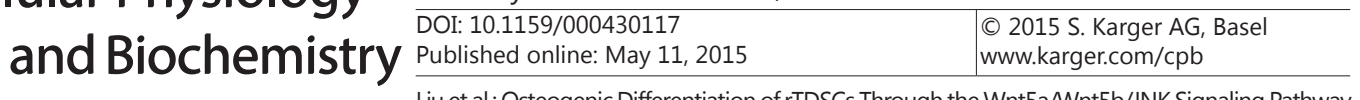

\section{Abbreviations}

TSCs (tendon stem cells); UMT (uniaxial mechanical tension); rTDSCs (rat tendonderived stem cells); MSCs (mesenchymal stem cells); GM (growth medium); DMEM (Dulbecco's Modified Eagle Medium); Osteo-M (osteogenic media); NC (non-loading control); AM (anisomycin).

\section{Acknowledgments}

This work was supported by the National Natural Science Foundation of China (grant no. 81230040).

\section{Disclosure Statement}

None declared by any author.

\section{References}

1 Kannus P, Jozsa L: Histopathological changes preceding spontaneous rupture of a tendon. A controlled study of 891 patients. J Bone Joint Surg Am 1991;73:1507-1525.

2 Bi Y, Ehirchiou D, Kilts TM, Inkson CA, Embree MC, Sonoyama W, Li L, Leet AI, Seo BM, Zhang L, Shi S, Young MF: Identification of tendon stem/progenitor cells and the role of the extracellular matrix in their niche. Nat Med 2007;13:1219-1227.

3 Gulotta LV, Chaudhury S, Wiznia D: Stem cells for augmenting tendon repair. Stem Cells Int DOI: $10.1155 / 2012 / 291431$.

4 Tao X, Liu J, Chen L, Zhou Y, Tang K: EGR1 Induces Tenogenic Differentiation of Tendon Stem Cells and Promotes Rabbit Rotator Cuff Repair. Cell Physiol Biochem 2015;35:699-709.

5 Chen L, Liu JP, Tang KL, Wang Q Wang GD, Cai XH, Liu XM: Tendon derived stem cells promote platelet-rich plasma healing in collagenase-induced rat achilles tendinopathy. Cell Physiol Biochem 2014;34:2153-2168.

6 Zhang J, Wang JH: Mechanobiological response of tendon stem cells: implications of tendon homeostasis and pathogenesis of tendinopathy. J Orthop Res 2010;28:639-643.

7 Hibi M, Lin A, Smeal T, Minden A, Karin M: Identification of an oncoprotein-and UV-responsive protein kinase that binds and potentiates the c-Jun activation domain. Gene Dev 1993;7:2135-2148.

8 Dérijard B, Hibi M, Wu IH, Barrett T, Su B, Deng T, Karin M, Davis RJ: JNK1: a protein kinase stimulated by UV light and Ha-Ras that binds and phosphorylates the c-Jun activation domain. Cell 1994;76:1025-1037.

9 Zhang X, Zhu J, Yang GY, Wang QJ, Qian L, Chen YM, Chen F, Tao Y, Hu HS, Wang T, Luo ZG: Dishevelled promotes axon differentiation by regulating atypical protein kinase C. Nat Cell Biol 2007;9:743-754.

10 Salinas PC: Modulation of the microtubule cytoskeleton: a role for a divergent canonical Wnt pathway. Trends Cell Biol 2007;17:333-342.

11 Liu F, Kohlmeier S, Wang CY: Wnt signaling and skeletal development. Cell Signal 2008;20:999-1009.

12 Mu C, Lv T, Wang Z, Ma S, Ma J, Liu J, Yu J, Mu J: Mechanical Stress Stimulates the Osteo/Odontoblastic Differentiation of Human Stem Cells from Apical Papilla via ERK 1/2 and JNK MAPK Pathways. Biomed Res Int DOI:10.1155/2014/494378.

13 Kwon HS, Johnson TV, Tomarev SI: Myocilin stimulates osteogenic differentiation of mesenchymal stem cells through mitogen-activated protein kinase signaling. J Biol Chem 2013;288:16882-16894.

14 Heidari N, Miller AV, Hicks MA, Marking CB, Harada H: Glucocorticoid-mediated BIM induction and apoptosis are regulated by Runx2 and c-Jun in leukemia cells. Cell Death Dis 2012; 3: e349.

15 Lee JS, Yi JK, An SY, Heo JS: Increased osteogenic differentiation of periodontal ligament stem cells on polydopamine film occurs via activation of integrin and PI3K signaling pathways. Cell Physiol Biochem 2014;34:1824-1834. 


\section{Cellular Physiology Cell Physiol Biochem 2015;36:517-530 \begin{tabular}{ll|l} 
and Biochemistry $10.1159 / 000430117$ & $\begin{array}{l}\text { (c) 2015 S. Karger AG, Basel } \\
\text { www.karger.com/cpb }\end{array}$ \\
\cline { 2 - 3 }
\end{tabular}

16 Qiao W, Chen L, Zhang M: MicroRNA-205 regulates the calcification and osteoblastic differentiation of vascular smooth muscle cells. Cell Physiol Biochem 2014;33:1945-1953.

17 Tetsunaga T, Nishida K, Furumatsu T, Naruse K, Hirohata S, Yoshida A, Saito T, Ozaki T: Regulation of mechanical stress-induced MMP-13 and ADAMTS- 5 expression by RUNX- 2 transcriptional factor in SW1353 chondrocyte-like cells. Osteoarthr Cartilage 2011;19:222-232.

18 Mbalaviele G, Sheikh S, Stains JP, Salazar VS, Cheng SL, Chen D, Civitelli R: Beta-catenin and bmp-2 synergize to promote osteoblast differentiation and new bone formation. J Cell Biochem 2005;94:403-418.

19 Johnson ML, Kamel MA: The wnt signaling pathway and bone metabolism. Curr Opin Rheumatol 2007;19:376-382.

20 Rong S, Zhao X, Jin X, Zhang Z, Chen L, Zhu Y, Yuan W: Vascular calcification in chronic kidney disease is induced by bone morphogenetic protein- 2 via a mechanism involving the $\mathrm{Wnt} / \beta$-catenin pathway. Cell Physiol Biochem 2014;34:2049-2060.

21 Bovolenta P, Rodriguez J, Esteve P: Frizzled/RYK mediated signalling in axon guidance. Development 2006;133:4399-4408.

22 Seifert JRK, Mlodzik M: Frizzled/PCP signalling: a conserved mechanism regulating cell polarity and directed motility. Nat Rev Genet 2007;8:126-138.

23 Wang Y, Nathans J: Tissue/planar cell polarity in vertebrates: new insights and new questions. Development 2007;134:647-658.

24 Ichida M, Yui Y, Yoshioka K, Tanaka T, Wakamatsu T, Yoshikawa H, Itoh K: Changes in cell migration of mesenchymal cells during osteogenic differentiation. Febs lett 2011;585:4018-4024.

25 Chai L, Cao C, Bi S, Dai X, Gan L, Guo R, Li S: Small Rho GTPase Racl determines human epidermal stem cell fate in vitro. Int J Mol Med 2010;25:723-727.

26 Schambony A, Wedlich D: Wnt5A/Ror2 regulate expression of XPAPC through an alternative noncanonical signaling pathway. Dev Cell 2007;12:779-792.

27 Randall RM, Shao YY, Wang L, Ballock RT: Activation of Wnt Planar cell polarity (PCP) signaling promotes growth plate column formation in vitro. J Orthop Res 2012;30:1906-1914.

28 Santiago F, Oguma J, Brown AM, Laurence J: Noncanonical Wnt signaling promotes osteoclast differentiation and is facilitated by the human immunodeficiency virus protease inhibitor ritonavir. Biochem Bioph Res Co 2012;417:223-230.

29 Oishi I, Suzuki H, Onishi N, Takada R, Kani S, Ohkawara B, Koshida I, Suzuki K, Yamada G, Schwabe GC, Mundlos S, Shibuya H, Takada S, Minami Y: The receptor tyrosine kinase Ror2 is involved in non-canonical Wnt5a/JNK signalling pathway. Genes Cells 2003;8:645-654.

30 Park KH, Kang JW, Lee EM, Kim JS, Yun RH, Kim M, Jeong SJ, Park YG, Kim SH: Melatonin promotes osteoblastic differentiation through the BMP/ERK/Wnt signaling pathways.J Pineal Res 2011;51:187-194.

31 Charoenpanich A, Wall ME, Tucker CJ, Andrews DM, Lalush DS, Dirschl DR, Loboa EG: Cyclic tensile strain enhances osteogenesis and angiogenesis in mesenchymal stem cells from osteoporotic donors. Tissue Eng Part A 2013;20:67-78.

32 Liu J, Chen L, Tao X, Tang K: Phosphoinositide 3-kinase/Akt signaling is essential for prostaglandin E2induced osteogenic differentiation of rat tendon stem cells. Biochem Bioph Res Co 2013;435:514-519.

33 Lin X, Yu Y, Zhao H, Zhang Y, Manela1 J, Tonetti DA: Overexpression of PKC $\alpha$ is required to impart estradiol inhibition and tamoxifen-resistance in a T47D human breast cancer tumor model. Carcinogenesis 2006;27:1538-1546.

34 Zielske SP, Stevenson M: Importin 7 may be dispensable for human immunodeficiency virus type 1 and simian immunodeficiency virus infection of primary macrophages. J Virol 2005;79:11541-11546.

35 Qiu Z, Huang C, Sun J, Qiu W, Zhang J, Li H, Jiang T, Huang K, Cao J: RNA interference-mediated signal transducers and activators of transcription 3 gene silencing inhibits invasion and metastasis of human pancreatic cancer cells. Cancer Sci 2007;98:1099-1106.

36 Wang J: Mechanobiology of tendon. J Biomech 2006;39:1563-1582.

37 Zhang J, Wang J: Production of PGE2 increases in tendons subjected to repetitive mechanical loading and induces differentiation of tendon stem cells into non-tenocytes. J Orthop Res 2010;28:198-203.

38 Lui P, Chan KM: Tendon-derived stem cells (TDSCs): from basic science to potential roles in tendon pathology and tissue engineering applications. Stem Cell Rev 2011;7:883-897. 


\section{Cellular Physiology Cell Physiol Biochem 2015;36:517-530 \begin{tabular}{ll|l} 
and Bioch 10.1159/000430117 & $\begin{array}{l}\text { O 2015 S. Karger AG, Basel } \\
\text { www.karger.com/cpb }\end{array}$ \\
\hline
\end{tabular}}

39 Shi Y, Li H, Zhang X, Fu Y, Huang Y, Lui PP, Tang T, Dai K: Continuous cyclic mechanical tension inhibited Runx2 expression in mesenchymal stem cells through RhoA-ERK1/2 pathway. J Cell Physiol 2011;226:2159-2169.

40 Shi Y, Fu, Y, Tong W, Geng Y, Lui PP, Tang T, Zhang X, Da K: Uniaxial mechanical tension promoted osteogenic differentiation of rat tendon-derived stem cells (rTDSCs) via the Wnt5a-RhoA pathway. J Cell Biochem 2012;113:3133-3142.

41 Bain J, Mclauchlan H, Elliott M, Cohen P: The specificities of protein kinase inhibitors: an update. Biochem J 2003;371:199-204.

42 Tang M, Peng Z, Mai Z, Chen L, Mao Q Chen Z, Chen Q, Liu L, Wang Y, Ai H: Fluid Shear Stress Stimulates Osteogenic Differentiation of Human Periodontal Ligament Cells via the Extracellular Signal-Regulated Kinase 1/2 and p38 Mitogen-Activated Protein Kinase Signaling Pathways. J Periodontol 2014;85:18061813.

43 Huang RL, Yuan Y, Tu J, Zou GM, Li Q: Opposing TNF- $\alpha /$ IL-1 $\beta$ and BMP-2-activated MAPK signaling pathways converge on Runx2 to regulate BMP-2-induced osteoblastic differentiation. Cell Death Dis 2014;5:e1187.

44 Zhang X, Ting K, Bessette CM, Culiat CT, Sung SJ, Lee H, Chen F, Shen J, Wang JJ, Kuroda S, Soo Chia: Nell-1, a key functional mediator of Runx2, partially rescues calvarial defects in Runx2+/- mice. J Bone Miner Res 2011;26:777-791.

45 Ahn HJ, Lee WJ, Kwack K, Kwon YD: FGF2 stimulates the proliferation of human mesenchymal stem cells through the transient activation of JNK signaling. Febs Lett 2009;583:2922-2926.

46 Glossop JR, Cartmell SH: Tensile strain and magnetic particle force application do not induce MAP3K8 and IL-1B differential gene expression in a similar manner to fluid shear stress in human mesenchymal stem cells. J Tissue Eng Regen M 2010;4:577-579.

47 Wu Y, Zhang X, Zhang P, Fang B, Jiang L: Intermittent traction stretch promotes the osteoblastic differentiation of bone mesenchymal stem cells by the ERK1/2-activated Cbfa1 pathway. Connect Tissue Res 2012;53:451-459.

48 Habas R, Dawid IB, He X: Coactivation of Rac and Rho by Wnt/Frizzled signaling is required for vertebrate gastrulation. Gene dev 2003;17:295-309.

49 Li L, Yuan H, Xie W, Mao J, Caruso AM, Mcmahon A, Sussman DJ, Wu D: Dishevelled proteins lead to two signaling pathways Regulation of LEF-1 and c-Jun N-terminal kinase in mammalian cells. J Biol Chem 1999;274:129-134.

50 Boutros M, Paricio N, Strutt DI, Mlodzik M: Dishevelled Activates JNK and Discriminates between JNK Pathways in Planar Polarity and wingless Signaling. Cell 1998;94:109-118.

51 Strutt DI, Weber U, Mlodzik M: The role of RhoA in tissue polarity and Frizzled signalling. Nature 1997;387:292-295.

52 Wang C, Zhao Y, Su Y, Li R, Lin Y, Zhou X, Ye L: C-Jun N-terminal kinase (JNK) mediates Wnt5a-induced cell motility dependent or independent of RhoA pathway in human dental papilla cells. PloS one 2013;8:e69440.

53 James AW, Pang S, Askarinam A, Corselli M, Zara JN, Goyal R, Chang L, Pan A, Shen J, Yuan W, Stoker D, Zhang X, Adams JS, Ting Kang, Soo Chia: Additive effects of sonic hedgehog and Nell-1 signaling in osteogenic versus adipogenic differentiation of human adipose-derived stromal cells. Stem Cells Dev 2012;21:2170-2178.

54 Pei L, Tontonoz P: Fat's loss is bone's gain. J Clin Invest 2004;113:805-806. 